\title{
Role of pentraxin-3 in risk assessment of patients with metabolic syndrome
}

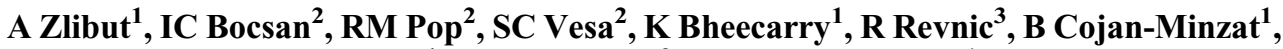 \\ S Lupu ${ }^{4}$, AD Buzoianu², L Agoston-Coldea ${ }^{1}$ \\ ${ }^{1}$ 2nd Department of Internal Medicine, Iuliu Hatieganu University of Medicine and Pharmacy, \\ Cluj-Napoca, Romania \\ ${ }^{2}$ Department of Pharmacology, Toxicology and Clinical Pharmacology, Iuliu Hatieganu University of Medicine \\ and Pharmacy, Cluj-Napoca, Romania \\ ${ }^{3}$ Department of Community Medicine, Iuliu Hatieganu University of Medicine and Pharmacy, \\ Cluj-Napoca, Romania \\ ${ }^{4}$ Department of Physiology, University of Medicine and Pharmacy, Târgu Mureş, Romania
}

Received: July 3, 2018

Accepted: June 19, 2019

Background: Inflammation plays a major role in the development of metabolic syndrome (MetS) and its progression. Recent studies have shown that pentraxin-3 (PTX-3), osteoprogerin (OPG), and tumor necrosis factor-alpha (TNF- $\alpha$ ) are key factors in MetS pathophysiology, but evidence for endorsing their clinical use is currently unclear and insufficient. Aim: The study aimed to evaluate the association between the inflammatory biomarkers' levels and the severity of MetS. Methods: The study was observational, transversal, prospective, cohort, and analytical type. We enrolled 80 patients $(\mathrm{M}: \mathrm{F}=1$, mean age $=55 \pm 10.77$ years $)$ who met MetS criteria. The study protocol included: medical history, physical examination, 6-min walk test distance (6MWTD), biochemical tests, electrocardiogram, echocardiography, and carotid ultrasonography. We also performed plasmatic measurement of PTX-3, OPG, and TNF- $\alpha$, in addition to standard biochemical tests. Results: Subjects with severe MetS had higher values of body mass index (BMI) and waist circumference $(p<0.001, p=0.001)$. PTX-3 levels were significantly higher in patients with severe MetS $(p=0.03)$ and the values were not influenced by age or gender. OPG positively correlated with BMI $(r=0.264, p=0.018)$. 6MWTD was lower in patients with severe MetS $(p=0.005)$, whereas CCA-IMT was higher in this group of patients $(p=0.005)$. In addition, the receiver operating characteristic (ROC) curve analysis for PTX-3 identified a cut-off value of $10.7 \mathrm{ng} / \mathrm{dl}$ that differentiates between mild and severe MetS [AUC 0.656 ; sensitivity = $47.1 \%(95 \% \mathrm{CI}=36.1 \%-62.3 \%)$; specificity $=78.9 \%(95 \% \mathrm{CI}=54.4 \%-93.9 \%)$ ]. Conclusion: PTX-3 was correlated with the severity of MetS, with other inflammatory parameters and cardiovascular tests. CCA-IMT and 6MWTD are useful in differentiating between mild and severe MetS.

Keywords: pentraxin-3, osteoprotegerin, tumor necrosis factor-alpha, metabolic syndrome, atherosclerosis

\section{Introduction}

Metabolic syndrome (MetS) represents a cluster of risk factors, including hypertension, dyslipidemia, elevated plasma glucose, and central obesity $(7,25)$, with a prevalence of $20 \%-25 \%$ in adults (7). MetS increases cardiovascular morbidity twofold, which is a major healthcare burden.

Corresponding author: Ioana Corina Bocsan, $\mathrm{MD}, \mathrm{PhD}$

Department of Pharmacology, Toxicology and Clinical Pharmacology,

Iuliu Hatieganu University of Medicine and Pharmacy

Marinescu str, no. 23, Cluj-Napoca, Romania

Phone: +40 740064 047; Fax: +40 264595 629; E-mails: corinabocsan@yahoo.com; bocsan.corina@umfcluj.ro 
The pathophysiological mechanisms behind the development and progression of MetS are not fully understood. Inflammation (14) and endothelial dysfunction (18) seem to play a significant role, leading to atherosclerosis and its complications, including cardiac ischemic disease (14). MetS is characterized by a chronic low-grade inflammation that involves inner humoral and cellular immunity (17). Recent studies have shown that some inflammatory cytokines, adipokines, neuropeptides, prothrombotic, and other factors are involved in MetS pathogenesis $(1,17,18)$. These mediators could be used to diagnose, monitor, or predict the severity of MetS.

Pentraxin-3 (PTX-3), like C-reactive protein (CRP), is a member of the pentraxin superfamily, which is produced by different cells in response to several inflammatory stimuli, including tumor necrosis factor-alpha (TNF- $\alpha$ ) and interleukin-1 (16). PTX-3 is released by monocytes/macrophages and endothelial cells and, to a lesser extent, by smooth muscle cells. It is overexpressed in atherosclerotic plaques (19), suggesting that it could be a valuable biomarker of atherosclerosis. A recent study reported that the plasmatic levels of PTX-3 were higher in subjects with MetS than in those without it. PTX-3 is also strongly correlated with metabolic parameters $(10,29)$, so it could be considered a marker for the diagnosis of MetS.

Osteoprotegerin (OPG) is a cytokine receptor belonging to the TNF receptor superfamily. OPG modulates the progression of the calcification of atherosclerotic lesions by acting as a decoy receptor for the receptor activator of nuclear factor kappa B ligand (4). The plasmatic level of OPG is significantly higher in patients with MetS compared to those without MetS, and OPG levels are correlated with the presence of microvascular lesions (27).

TNF- $\alpha$ is involved in the progression of atherosclerotic plaques (3), due to an increased uptake of atherogenic lipoproteins by enhanced scavenger receptor activity (22). Current research confirms the involvement of TNF- $\alpha$ in the pathogenesis of MetS $(3,23)$.

Thus, PTX-3, OPG, and TNF- $\alpha$ are all connected with the inflammatory process within subclinical atherosclerosis $(4,10,23,27,29)$ and they were investigated as possible markers of cardiovascular disorders with an inflammatory component. Their combined use in the assessment of MetS severity and subclinical atherosclerotic lesions has not yet been addressed.

The aim of this study was to assess the association between these inflammatory biomarkers, the severity of MetS, and cardiovascular risk.

\section{Materials and Methods}

\section{Patients}

The study was observational, transversal, prospective, cohort, analytical type. We enrolled 80 patients $(\mathrm{M}: \mathrm{F}=1$, mean age $=55 \pm 10.77$ years) who met MetS criteria (25). The patients were evaluated in First Department of Internal Medicine of County Hospital, between March 2016 and 2017. The study protocol is in conformity with the Declaration of Helsinki and was approved by the Ethics Committee of the University of Medicine and Pharmacy. All patients were informed about the study protocol and signed the informed consent.

MetS was defined by the presence of three or more of the following criteria: waist circumference $>94 \mathrm{~cm}$ in men/ $>80 \mathrm{~cm}$ in women; systolic blood pressure (SBP) over $130 \mathrm{mmHg}$ or/and diastolic blood pressure (DBP) over $85 \mathrm{mmHg}$, or antihypertensive treatment; triglycerides $>150 \mathrm{mg} / \mathrm{dl}$; HDL cholesterol $<40 \mathrm{mg} / \mathrm{dl}$ in $\mathrm{men} /<50 \mathrm{mg} / \mathrm{dl}$ in women; fasting serum glucose $>100 \mathrm{mg} / \mathrm{dl}$ or confirmed diabetes mellitus (7). 
The exclusion criteria were: inflammatory disorders, coronary artery disease, impaired renal function or retinopathy, females under oral contraceptives, patients undergoing treatment with vitamin $\mathrm{K}$ antagonists, and patients with poor echocardiographic window or those who refused to participate in the research.

The investigation protocol included: medical history, physical examination, 6-minute walk test (6MWT), biochemical tests, electrocardiography, echocardiography, and carotid ultrasonography.

Waist size, SBP, and DBP were measured on admission. Body mass index (BMI) was calculated to diagnose and grade obesity [overweight $\left(25-29 \mathrm{~kg} / \mathrm{m}^{2}\right)$ or obese $\left(\geq 30 \mathrm{~kg} / \mathrm{m}^{2}\right)$ ]. Patients who smoked at least 1 cigarette/day within the previous 2 months were considered active smokers. Impaired fasting glucose was defined by an elevated fasting plasma glucose concentration ( $>100$ and $<126 \mathrm{mg} / \mathrm{dl}$ ). We recorded the history of hypertension, diabetes mellitus, dyslipidemia, and current treatment.

\section{Biochemical analysis}

Standard biochemical markers were measured in the Department of Biochemistry of County Hospital using a Beckman Coulter AU480 Chemistry Analyzer (Atlanta, GA, USA). The inflammatory markers were determined using ELISA methods with specific standardized kits: high-sensitivity CRP (hs-CRP; DIAsource Immuno Assays SA, Belgium), PTX-3 (OmniKineHuman, Assay biotech, Germany), OPG (Abbexa, USA), and TNF- $\alpha$ (DuoSet, R\&D Systems Europe). All the aforementioned determinations were assayed according to the manufacturers' instructions.

\section{Echocardiography}

Standard echocardiography was performed on a General Electric Logiq 7 (GE Medical Systems, Tokyo, Japan) echocardiograph with a M3S 2.5-3.5 MHz active matrix phased array transducer. The following measurements were recorded: left ventricle ejection fraction, end-diastolic volume, end-systolic volume, left ventricular mass, mitral flow pulsed wave Doppler (E wave, A wave, E/A ratio, and deceleration time), tissue Doppler imaging measurements, $\mathrm{E} / \mathrm{E}^{\prime}$ ratio, and left atrial volume; also in addition, pulmonary artery pressure and tricuspid annular plane systolic excursion were determined. These parameters were evaluated to exclude any ischemic impairment of the heart.

\section{Carotid ultrasonography}

Common carotid arteries (CCAs) were examined on a General Electric Logiq 7 (GE Medical Systems, Japan) device with a $7.5 \mathrm{MHz}$ linear array transducer. CCA diameters were measured $10 \mathrm{~mm}$ proximal to the bulb, on longitudinal axis. CCA wall thickness was quantified based on the intima-media thickness (CCA-IMT), measured from the leading edges of the transition zones between lumen - intima and media - adventitia. Atherosclerotic plaques were defined as focal wall regions $>1.5-\mathrm{mm}$ thick that protruded into the lumen (12).

\section{Arterial stiffness}

Arterial wall stiffness was assessed by two estimates of the arterial stiffness index by M-mode ultrasonography and calculated by the formula: $\log (\mathrm{SBP} / \mathrm{DBP}) /[(\mathrm{Ds}-\mathrm{Dd}) / \mathrm{Dd}]$, where Ds and Dd are the end-systolic and end-diastolic diameters of the CCA, respectively and SBP and DBP are the systolic and diastolic blood pressures (11). Carotid femoral pulse wave 
velocity (PWV) was measured in the supine position using an automatic waveform analyzer (TensioMed Ltd., Hungary).

\section{Statistical analysis}

Statistical analysis was performed using the MedCalc Software, version 16.1.2 (Belgium). Parametric and non-parametric distribution of variables was assessed by the KolmogorovSmirnov test. Parametric data were expressed as mean \pm standard deviation and non-parametric data were expressed as median value and interquartile ranges. Relative frequencies for categorical variables were reported as percentages. Correlations between parameters were determined by the Pearson's test for parametric data and by Spearman's test for non-parametric data. We used the $\chi^{2}$ test for data analysis. The results were considered statistically significant if $p<0.05$.

\section{Results}

\section{The demographic and clinical data}

Demographic and clinical data are presented in Table I.

To establish the severity of MetS, the patients were divided in two groups: the first group included the patients with minimally three components of MetS and they were considered having the mild form of MetS, whereas the second group included patients with the severe form of MetS presenting four or five diagnostic criteria. Nineteen patients (23.75\%) had mild MetS, whereas 61 patients (76.25\%) presented severe MetS. The severity of MetS was not correlated with the patients' gender $(p>0.05)$.

There was no correlation between anthropometric parameters and patients' age or gender $(p>0.05)$.

More patients with severe MetS were smokers as compared to the group with mild MetS (31.6\% vs. $49.2 \%)$, but the difference was not significant $(p>0.05)$.

Patients with severe MetS had significantly higher values of BMI, body surface area, and waist circumference compared to patients with mild MetS (Table I).

\section{Biological evaluation}

There was a weak negative correlation between HDL-cholesterol and patients' BMI $(r=-0.205, p=0.05)$, but not between the former and waist circumference $(r=-0.191$, $p=0.09$ ). The levels of biological parameters were not influenced by the patients' age or gender.

HDL-cholesterol was significantly lower, whereas triglycerides were significantly higher in patients with severe MetS (Table II). The other biological parameters, which are not components of MetS, did not differ between mild and severe MetS $(p>0.05)$.

Regarding the inflammatory biomarkers, only PTX-3 had significantly higher values in patients with severe MetS (Table II). The level of PTX-3 was not influenced by the patients' age or gender $(p>0.05)$.

Regarding the association of the inflammatory biomarkers with anthropometric and biological parameters, the only positive correlation was that of OPG levels with patients' BMI $(r=0.264, p=0.018)$. PTX-3 and TNF- $\alpha$ were not correlated with BMI. None of the inflammatory parameters were correlated with waist circumference or with other biological parameters $(p>0.05)$. 
Table I. Demographic and clinical data

\begin{tabular}{|c|c|c|c|}
\hline Characteristics & No. of MetS components & Median values & $p$ \\
\hline \multirow[t]{2}{*}{ Age (years) } & 3 & $50.18(43.53-58.72)$ & \multirow[t]{2}{*}{0.089} \\
\hline & $>3$ & $57.31(45.84-65.20)$ & \\
\hline \multirow[t]{2}{*}{ Gender (M/F) (\%) } & 3 & $47.4 / 52.6$ & \multirow[t]{2}{*}{1} \\
\hline & $>3$ & $50.8 / 49.2$ & \\
\hline \multirow[t]{2}{*}{ Body surface area $\left(\mathrm{m}^{2}\right)$} & 3 & $1.55(1.42-1.69)$ & \multirow[t]{2}{*}{0.002} \\
\hline & $>3$ & $1.75(1.62-1.90)$ & \\
\hline \multirow[t]{2}{*}{ Body mass index (BMI; kg/m²) } & 3 & $28.90(27.39-32.36)$ & \multirow[t]{2}{*}{$<0.001$} \\
\hline & $>3$ & $34.52(31.01-37.28)$ & \\
\hline \multirow[t]{2}{*}{ Waist circumference $(\mathrm{cm})$} & 3 & $101(98-110)$ & \multirow[t]{2}{*}{0.001} \\
\hline & $>3$ & $112(102-124.5)$ & \\
\hline \multirow[t]{2}{*}{ Systolic blood pressure (mmHg) } & 3 & $160(140-180)$ & \multirow[t]{2}{*}{0.7} \\
\hline & $>3$ & $160(150-171)$ & \\
\hline \multirow[t]{2}{*}{ Diastolic blood pressure ( $\mathrm{mmHg}$ ) } & 3 & $90(90-110)$ & \multirow[t]{2}{*}{0.7} \\
\hline & $>3$ & $95(90-100)$ & \\
\hline
\end{tabular}

Data are expressed as median; 25-75th percentile. Bold values represent statistical significant $(p<0.05)$. BMI: body mass index; F: female; M: male; MetS: metabolic syndrome

The plasmatic values of TNF- $\alpha$ had a moderate positive correlation with PTX-3 $(r=0.395, p<0.001)$ and OPG $(r=0.221, p=0.049)$. The plasmatic levels of PTX-3, OPG, TNF- $\alpha$, and hs-CRP $(p>0.05)$ were not correlated.

\section{Cardiovascular evaluation}

6MWT was negatively correlated with BMI $(r=-0.457, p<0.001)$ and waist circumference $(r=-0.397, p<0.001)$. Regarding the inflammatory biomarkers, only PTX-3 was negatively correlated with 6MWT $(r=-0.255, p=0.02)$.

The measured distance in 6MWT was significantly lower in patients with severe MetS as compared to the mild MetS group $(p=0.005)$. Thus, the severe MetS group had 48 patients (78.7\%) with the 6-min walk test distance (6MWTD) below 610, whereas the mild MetS group had only 8 patients $(42.8 \% ; p=0.004)$. The distance of $610 \mathrm{~m}$ was positively correlated with a higher number of MetS components $(p=0.004)$.

CCA-IMT was positively correlated with the patients' BMI $(r=0.302, p=0.006)$. Moreover, both CCA-IMT and PWV were positively correlated with waist circumference ( $r=0.374, p=0.001$ and $r=0.253, p=0.024$, respectively).

In addition, CCA-IMT was increased in patients with severe MetS (Table III). Forty-three patients (70.5\%) with severe MetS presented a CCA-IMT above $0.8 \mathrm{~mm}$ as compared to seven patients $(36.8 \%)$ with mild MetS $(p=0.014)$.

None of the investigated inflammatory biomarkers were correlated with CCA-IMT or $\operatorname{PWV}(p>0.05)$. 
Table II. Laboratory values

\begin{tabular}{|c|c|c|c|}
\hline Characteristics & No. of MetS components & Median values & $p$ \\
\hline \multirow[t]{2}{*}{ Fasting plasma glucose $(\mathrm{mg} / \mathrm{dl})$} & 3 & $99(88-131)$ & \multirow[t]{2}{*}{0.09} \\
\hline & $>3$ & $118(104-134)$ & \\
\hline \multirow[t]{2}{*}{ Triglyceridemia (mg/dl) } & 3 & $178(137-217)$ & \multirow[t]{2}{*}{0.05} \\
\hline & $>3$ & $199(159.5-270)$ & \\
\hline \multirow[t]{2}{*}{ HDL-cholesterol (mg/dl) } & 3 & $42(38-48)$ & \multirow[t]{2}{*}{$<0.001$} \\
\hline & $>3$ & $36(33-39)$ & \\
\hline \multirow[t]{2}{*}{ HS-C reactive protein $(\mathrm{ng} / \mathrm{ml})$} & 3 & $0.92(0.71-1.41)$ & \multirow[t]{2}{*}{0.195} \\
\hline & $>3$ & $0.85(0.595-1.11)$ & \\
\hline \multirow[t]{2}{*}{ Total cholesterol (mg/dl) } & 3 & $201(175-235)$ & \multirow[t]{2}{*}{0.95} \\
\hline & $>3$ & $210(173.5-234.5)$ & \\
\hline \multirow[t]{2}{*}{ LDL-cholesterol (mg/dl) } & 3 & $129.2(100.6-155.4)$ & \multirow[t]{2}{*}{0.892} \\
\hline & $>3$ & $126(88.4-149.8)$ & \\
\hline \multirow[t]{2}{*}{ Creatinine (mg/dl) } & 3 & $0.79(0.68-0.91)$ & \multirow[t]{2}{*}{0.87} \\
\hline & $>3$ & $0.86(0.75-0.995)$ & \\
\hline \multirow[t]{2}{*}{ PTX-3 (ng/dl) } & 3 & $0.36(0.36-10.76)$ & \multirow[t]{2}{*}{0.030} \\
\hline & $>3$ & $10.04(0.36-82.29)$ & \\
\hline \multirow[t]{2}{*}{ OPG (pg/dl) } & 3 & $306.66(244.84-504.49)$ & \multirow[t]{2}{*}{0.15} \\
\hline & $>3$ & $430.30(283.48-572.49)$ & \\
\hline \multirow[t]{2}{*}{ TNF- $\alpha(\mathrm{pg} / \mathrm{dl})$} & 3 & $557.75(3.90-3,188.37)$ & \multirow[t]{2}{*}{0.53} \\
\hline & $>3$ & $1186.81(59.40-3,359.94)$ & \\
\hline
\end{tabular}

Data are expressed as median; 25-75th percentile. Bold values represent statistical significant $(p<0.05)$. HDL-cholesterol: high-density lipoprotein cholesterol; hs-CRP: high-sensitivity C-reactive protein; LDL-cholesterol: low-density lipoprotein cholesterol; MetS: metabolic syndrome; PTX-3: pentraxin-3; OPG: osteoprotegerin; TNF- $\alpha$ : tumor necrosis factor-alpha.

ROC curve analysis for assessment of sensibility and specificity of the studied biomarkers in MetS severity assessment

The calculated PTX-3 cut-off value $(10.7 \mathrm{ng} / \mathrm{dl})$ differentiated between mild and severe MetS $[\mathrm{AUC}=0.656$; sensitivity $(\mathrm{Se})=47.1 \%(95 \% \mathrm{CI}=36.1 \%-62.3 \%)$; specificity $(\mathrm{Sp})=78.9 \%$ $(95 \% \mathrm{CI}=54.4 \%-93.9 \%)]$. Regarding OPG and TNF- $\alpha$, receiver operating characteristic (ROC) analysis did not reach statistical significance.

In addition, the ROC curve for 6MWT and CCA-IMT were analyzed. Accordingly, the 6MWTD had a calculated cut-off value of $610 \mathrm{~m}$, which made a significant difference between mild and severe MetS [AUC 0.712; Se=78.69\% (95\% CI =66.3\%-88.1\%); $\mathrm{Sp}=57.9 \%(95 \% \mathrm{CI}=33.5 \%-79.7 \%)]$. CCA-IMT had an AUC of 0.675 for a cut off 
Table III. Cardiovascular parameters

\begin{tabular}{|c|c|c|c|}
\hline Characteristics & No. of MetS components & Median values & $p$ \\
\hline \multirow[t]{2}{*}{ 6MWDT (m) } & 3 & $670(700-560)$ & \multirow[t]{2}{*}{0.005} \\
\hline & $>3$ & $560(450-600)$ & \\
\hline \multirow[t]{2}{*}{ Carotid stiffness index $\beta$} & 3 & $12.88(-14.81-16.28)$ & \multirow[t]{2}{*}{0.982} \\
\hline & $>3$ & $12.62(-11.09-14.93)$ & \\
\hline \multirow[t]{2}{*}{ Pulse wave velocity $(\mathrm{m} / \mathrm{s})$} & 3 & $10.72(6.02-12.22)$ & \multirow[t]{2}{*}{0.415} \\
\hline & $>3$ & $10.84(9.45-12.224)$ & \\
\hline \multirow[t]{2}{*}{ CCA-IMT (mm) } & 3 & $0.8(0.7-0.9)$ & \multirow[t]{2}{*}{0.017} \\
\hline & $>3$ & $0.9(0.8-0.995)$ & \\
\hline
\end{tabular}

Data are expressed as median; 25-75th percentile. Bold values represent statistical significant $(p<0.05)$. 6MWTD: 6-min walk test distance; CCA-IMT: common carotid artery - intima media thickness; MetS: metabolic syndrome.

value of $0.8 \mathrm{~mm}$, with a sensitivity of $70.5 \%(95 \% \mathrm{CI}=57.4 \%-81.5 \%)$ and a specificity of $63.16 \%(95 \% \mathrm{CI}=38.4 \%-83.7 \%)$.

\section{Multivariate analysis of factors associated with MetS severity}

To find out which parameter was independently associated with the severity of MetS, several predictive models using multivariate logistic regression were constructed. The most stable included the following variables: 6MWT, PTX-3, and CCA-IMT (Table IV). Based on previous reported results (28), the PTX-3 values were adjusted according to the patients' age. Only 6MWTD $<610 \mathrm{~m}$ and CCA-IMT $>0.8 \mathrm{~mm}$ were independently linked to severe MetS, in both analyses with or without adjustment of PTX-3 values according to the patients' age.

PTX-3 was negatively correlated with the 6MWT $(r=-0.255 ; p=0.02)$, which can explain why it was not independently associated with severe MetS. The 6MWT had a much larger probability to be linked to severe MetS (OR=4.2 vs. 2.8).

Table IV. Multivariate analysis for the severity of MetS

\begin{tabular}{|l|c|c|c|c|}
\hline & \multicolumn{2}{|c|}{ Unadjusted for age } & \multicolumn{2}{c|}{ Adjusted for age } \\
\hline Variables & $\boldsymbol{p}$ & OR [95\% CI] & $\boldsymbol{p}$ & OR [95\% CI] \\
\hline $6 \mathrm{MWTD}>610 \mathrm{~m}$ & 0.016 & $4.246[1.305-13.816]$ & 0.024 & $4.668[1.228-17.740]$ \\
\hline PTX-3 $>10.7 \mathrm{ng} / \mathrm{dl}$ & 0.121 & $2.837[0.760-10.588]$ & 0.115 & $2.990[0.766-11.674]$ \\
\hline CCA-IMT $>0.8 \mathrm{~mm}$ & 0.020 & $4.027[1.244-13.031]$ & 0.020 & $4.209[1.253-14.140]$ \\
\hline
\end{tabular}

6MWTD: 6-min walk test distance; CCA-IMT: common carotid artery - intima media thickness; MetS: metabolic syndrome; PTX-3: pentraxin-3; CI: confidence interval; OR: odds ratio 


\section{Discussion}

This study describes the plasmatic profile of PTX-3, OPG, and TNF- $\alpha$ in patients with MetS, to establish their potential value for MetS risk assessment. Among the three biomarkers, PTX-3 had the highest correlation with the severity of MetS. It was found that PTX-3 plasmatic level was increased in patients with MetS, and a value higher than $10.7 \mathrm{ng} / \mathrm{dl}$ has a moderate sensitivity in the prediction of severe forms of the disease. In addition to this inflammatory biomarker, two other tests, 6MWT and CCA-IMT, were independently associated with severe MetS.

Inflammation in the vasculature is an important pathogenic link between cardiovascular diseases and MetS (26). Patients with MetS are more susceptible to develop diabetes mellitus, some cancers, and cardiovascular diseases (13) with an increased risk of morbidity and mortality. Since this syndrome consists of several cardiovascular risk factors, their combination could have a synergistic and multiplicative action, greatly amplifying the effects of each individual factor. It is often not possible to establish a cause-effect relationship between some risk factors and MetS and, sequentially, the cardiovascular risk. A better stratification of MetS' severity may allow us to understand which patients have a greater risk for cardiovascular events, leading to early introduction of therapeutic measures, whether pharmacological or not.

In this study, we included patients with only MetS, without clinical manifestation of atherosclerosis. Many studies have shown that there is a correlation between MetS and inflammatory mediators, including IL-6, TNF- $\alpha$, PTX-3, and hs-CRP $(10,13,21,23,29)$, but there are few data regarding their usage as biomarkers in the assessment of MetS' severity.

In this study, PTX-3 was correlated with MetS severity and its cut-off value of $10.7 \mathrm{ng} / \mathrm{dl}$ differentiated between mild and severe MetS. The same findings were reported by Karakas et al., who noticed that patients with severe MetS (five components) had higher values of PTX-3 compared to patients with mild or moderate MetS (three or four components) (9). Regarding hs-CRP, unlike this study, Karakas et al. reported that patients with severe MetS had higher hs-CRP as compared with mild MetS patients (9). We did not observe a different hs-CRP value in patients with severe as compared to mild MetS, or any correlation between the investigated inflammatory markers and hs-CRP. This result is similar to that reported by Miyazaki et al. (15) who, however, included patients with MetS and previous acute myocardial infarction.

The role of PTX-3 in atherosclerosis prompted us to evaluate whether a correlation exists between plasma PTX-3 and cardiometabolic risk factors in the syndrome. There was no correlation between PTX-3 and a specific component of MetS or other biological parameters. The results from the literature are controversial. Tabak et al. (24) found that PTX-3 is positively correlated with hs-CRP and LDL-cholesterol in patients with MetS. Yamasaki et al. (28) and Miyazaki et al. (15) showed that PTX-3 was inversely correlated with triglyceride levels and BMI in healthy subjects without MetS or associated cardiovascular diseases, and in patients with previous acute MI, respectively. Kardas et al. (10) and Zanetti et al. (29) showed that PTX-3 levels were positively correlated with triglycerides and negatively correlated with HDL-C levels in obese children and adolescents with MetS and cardiovascular diseases. A possible explanation of these controversial results consists in different inclusion criteria and different studied groups. It is possible that the relationships between PTX-3, atherosclerosis, and lipids are complex and are differently regulated under healthy and pathological conditions. 
PTX-3 dynamics changes over time in association with the presence of several cardiovascular risk factors or the presence of MetS components. This is based on the observation that PTX-3 is positively associated with other inflammatory markers, but not with the metabolic profile. Both OPG and PTX-3 positively correlated with TNF- $\alpha$. This was an expected result, knowing that PTX-3 secretion is stimulated by TNF- $\alpha$, whereas OPG belongs to the TNF- $\alpha$ superfamily $(4,19)$. Therefore, PTX-3 could become a marker to identify subclinical atherosclerosis, but further studies are needed to establish what its exact role is in the inflammatory process associated with cardiovascular disease.

Endothelial dysfunction plays an important role in the development of atherosclerosis, leading to changes in the vessel intima and an increase in arterial stiffness. CCA-IMT is known to be a parameter that identifies subclinical atherosclerosis. It has been reported that CCA-IMT is a predictor of CV events, independently of blood pressure, lipid profile, and glucose levels in patients with MetS $(8,29)$. In addition, it has been proven that CCA-IMT is associated with the progression of MetS over time (29). In this study, CCA-IMT was positively correlated with anthropometric parameters, but not with PTX-3 or OPG. For CCA-IMT, a cut-off value of $0.8 \mathrm{~mm}$ differentiated between the mild and moderate forms of MetS. These observations were different from the results reported in Zanetti et al.'s study (29). They described a positive association between CCA-IMT and PTX-3 plasmatic levels, but after adjustment for HDL-cholesterol, this association did not persist (29). The results from this study were similar to those of Baragetti et al. (2). They reported that PTX-3 was no longer correlated with CCA-IMT after adjustment for age, sex, hypertension, diabetes, lipid profiles, cardiovascular events, and medical therapy (2). In this study, PTX-3 was not correlated with CCA-IMT in either uni- or multivariate analysis, before or after adjustment for age. These findings suggest that in addition to PTX-3 other factors play an important role in promoting carotid artery media thickening in MetS, and the inflammatory status is not the only mechanism that leads to endothelial dysfunction and atherosclerosis progression.

6MWTD is a rapid and inexpensive method to assess functional exercise capacity in patients with cardiovascular diseases or MetS (5). In the present study, 6MWT was inversely correlated with BMI and waist circumference, reflecting a low physical activity in patients with obesity or overweight. The measured distance was significantly lower in patients with severe MetS. The analysis showed that a distance of $610 \mathrm{~m}$ may differentiate between the mild and severe forms of MetS. PTX-3 negatively correlated with 6MWT in univariate analysis, but after multivariate analysis only six MWT and CCA-IMT were independently linked to severe MetS. Rubinsztajn et al. (20) reported no changes of 6MWT in COPD patients with or without MetS, but the other inflammatory markers like IL-6 and CRP were higher in patients with MetS, suggesting that respiratory illness rather than subclinical inflammation may be responsible for reduced physical activity. In another study performed in patients with cardiac surgeries, PTX-3 as inflammatory marker showed a close association with 6MWT distance, similarly to our data (6).

The strength of this study is that we studied several biomarkers involved in MetS and we evaluated the relationship between each of them and anthropomorphic and biochemical variables. However, this study has some limitations. A relatively small number of patients were included in the study, which might diminish the strength of the presented data. In addition, patients' long-term follow-up would have provided more information regarding the progression of carotid plaques and investigated biomarkers. It might be interesting to evaluate the biomarkers over time to assess the disease progression. 


\section{Conclusions}

PTX-3 was correlated with the severity of MetS, with other inflammatory parameters and cardiovascular tests. CCA-IMT and 6MWTD are useful in differentiating between mild and severe MetS. PTX-3 could become a useful biomarker in the evaluation of subclinical atherosclerosis in patients with cardiometabolic risk factors and MetS, but further studies are needed to confirm its exact role in the inflammatory process associated with MetS.

\section{Acknowledgements}

This research was supported by the internal grant no. 4995/18/08.03.2016 of the "Iuliu Hatieganu" University of Medicine and Pharmacy, Cluj-Napoca, Romania.

\section{Conflict of interest}

The authors declare no conflict of interest.

\section{REFERENCES}

1. Alberti KG, Eckel RH, Grundy SM, Zimmet PZ, Cleeman JI, Donato KA, Fruchart JC, James WP, Loria CM, Smith SC Jr, International Diabetes Federation Task Force on Epidemiology and Prevention, National Heart, Lung, and Blood Institute, American Heart Association, World Heart Federation, International Atherosclerosis Society, International Association for the Study of Obesity: Harmonizing the metabolic syndrome: a joint interim statement of the International Diabetes Federation Task Force on Epidemiology and Prevention; National Heart, Lung, and Blood Institute; American Heart Association; World Heart Federation; International Atherosclerosis Society; and International Association for the Study of Obesity. Circulation 120, 1640-1645 (2009)

2. Baragetti A, Knoflach M, Cuccovillo I, Grigore L, Casula M, Garlaschelli K, Mantovani A, Wick G, Kiechl S, Willeit J, Bottazzi B, Catapano AL, Norata GD: Pentraxin 3 (PTX3) plasma levels and carotid intima media thickness progression in the general population. Nutr. Metab. Cardiovasc. Dis. 24, 518-523 (2014)

3. Boesten LS, Zadelaar AS, van Nieuwkoop A, Gijbels MJ, de Winther MP, Havekes LM, van Vlijmen BJ: Tumor necrosis factor-alpha promotes atherosclerotic lesion progression in APOE*3-Leiden transgenic mice. Cardiovasc. Res. 66, 179-185 (2005)

4. van Campenhout A, Golledge J: Osteoprotegerin, vascular calcification and atherosclerosis. Atherosclerosis 204, 321-329 (2009)

5. Eleutério-Silva MA, Sá da Fonseca LJ, Velloso EP, da Silva Guedes G, Sampaio WO, da Silva WF, Mota-Gomes MA, da Silva Lima LV, Santos RA, Rabelo LA: Short-term cardiovascular physical programme ameliorates arterial stiffness and decreases oxidative stress in women with metabolic syndrome. J. Rehabil. Med. 45, 572-579 (2013)

6. Ferratini M, Ripamonti V, Masson S, Grati P, Racca V, Cuccovillo I, Raimondi E, Capomolla S, Macchi C, Coruzzi P, Vago T, Calvo M, Mantovani A, Latini R: Pentraxin-3 predicts functional recovery and 1-year major adverse cardiovascular events after rehabilitation of cardiac surgery patients. J. Cardiopulm. Rehabil. Prev. 32, 17-24 (2012)

7. International Diabetes Federation (2006): IDF Consensus Worldwide Definition of Metabolic Syndrome, International Diabetes Federation, Brussels, MD. Available at: https://www.idf.org/e-library/consensusstatements/60-idfconsensus-worldwide-definitionof-the-metabolic-syndrome. Accessed on: 23 October 2017

8. Iglesias del Sol A, Bots ML, Grobbee DE, Hofman A, Witteman JCM: Carotid intima-media thickness at different sites: relation to incident myocardial infarction; the Rotterdam Study. Eur. Heart J. 23, 934-940 (2002)

9. Karakas MF, Buyukkaya E, Kurt M, Motor S, Akcay AB, Karakas E, Buyukkaya Ş, Sen N: Serum pentraxin-3 levels are associated with the severity of metabolic syndrome. Med. Princ. Pract. 61, 278-285 (2013)

10. Kardas F, Akın L, Kurtoglu S, Kendirci M, Kardas Z: Plasma pentraxin 3 as a biomarker of metabolic syndrome. Indian J. Pediatr. 82, 35-38 (2015)

11. Kawasaki T, Sasayama S, Yagi S, Asakawa T, Hirai T: Non-invasive assessment of the age related changes in stiffness of major branches of the human arteries. Cardiovasc. Res. 21, 678-687 (1987) 
12. Kim GH, Youn HJ: Is carotid artery ultrasound still useful method for evaluation of atherosclerosis? Korean Circ. J. 47, 1-8 (2017)

13. Lakka HM, Laaksonen DE, Lakka TA, Niskanen LK, Kumpusalo E, Tuomilehto J, Salonen JT: The metabolic syndrome and total and cardiovascular disease mortality in middle-aged men. JAMA 288, 2709-2716 (2002)

14. Mathieu P, Pibarot P, Despres JP: Metabolic syndrome: the danger signal in atherosclerosis. Vasc. Health Risk Manag. 2, 285-302 (2006)

15. Miyazaki T, Chiuve S, Sacks F, Ridker PM, Libby P, Aikawa M: Plasma pentraxin 3 levels reflect metabolic syndrome in patients with coronary artery disease. PLoS One. 9, e94073 (2014)

16. Moon YS, Kim DH, Song DK: Serum tumor necrosis factor-alpha levels and components of the metabolic syndrome in obese adolescents. Metabolism 53, 863-867 (2004)

17. Paragh G, Seres I, Harangi M, Fulop P: Dynamic interplay between metabolic syndrome and immunity. Adv. Exp. Med. Biol. 824, 171-190 (2014)

18. Picchi A, Gao X, Belmadani S, Potter BJ, Focardi M, Chilian WM, Zhang C: Tumor necrosis factor-alpha induces endothelial dysfunction in the prediabetic metabolic syndrome. Circ. Res. 99, 69-77 (2006)

19. Rolph MS, Zimmer S, Bottazzi B, Garlanda C, Mantovani A, Hansson GK: Production of the long pentraxin PTX3 in advanced atherosclerotic plaques. Arterioscler. Thromb. Vasc. Biol. 22, 10-14 (2002)

20. Rubinsztajn R, Przybyłowski T, Maskey-Warzęchowska M, Paplińska-Goryca M, Nejman-Gryz P, Karwat K, Chazan R: Metabolic syndrome as a factor affecting systemic inflammation in patients with chronic obstructive pulmonary disease. Adv. Exp. Med. Biol. 1021, 55-62 (2017)

21. Rudd JH, Myers KS, Bansilal S, Machac J, Woodward M, Fuster V, Farkouh ME, Fayad ZA: Relationships among regional arterial inflammation, calcification, risk factors, and biomarkers: a prospective fluorodeoxygucose positron-emission tomography/computed tomography imaging study. Circ. Cardiovasc. Imaging. 2, 107-115 (2009)

22. Schreyer SA, Peschon JJ, LeBoeuf RC: Accelerated atherosclerosis in mice lacking tumor necrosis factor receptor. J. Biol. Chem. 271, 26174-26178 (1996)

23. Srikanthan K, Feyh A, Visweshwar H, Shapiro JI, Sodhi K: Systematic review of metabolic syndrome biomarkers: a panel for early detection, management, and risk stratification in the West Virginian population. Int. J. Med. Sci. 13, 25-38 (2016)

24. Tabak O, Simsek G, Erdenen F, Sozer V, Hasoglu T, Gelisgen R, Altunoglu E, Muderrisoglu C, Senyigit A, Uzun $\mathrm{H}$ : The relationship between circulating irisin, retinol binding protein-4, adiponectin and inflammatory mediators in patients with metabolic syndrome. Arch. Endocrinol. Metab. 61, 515-523 (2017)

25. Talwalkar PG, Sreenivas CG, Gulati A, Baxi H: Journey in guidelines for lipid management: from adult treatment panel (ATP)-I to ATP-III and what to expect in ATP-IV. Indian J. Endocrinol. Metab. 17, 628-635 (2013)

26. Tanaka A, Shimad K, Sano T, Namba M, Sakamoto T, Nishida Y, Kawarabayashi T, Fukuda D, Yoshikawa J: Multiple plaque rupture and C-reactive protein in acute myocardial infarction. J. Am. Coll. Cardiol. 45, 1594-1599 (2005)

27. Tavintharan S, Pek LT, Liu JJ, Ng XW, Yeoh LY, Su Chi L, Chee Fang S: Osteoprotegerin is independently associated with metabolic syndrome and microvascular complications in type 2 diabetes mellitus. Diab. Vasc. Dis. Res. 11, 359-362 (2014)

28. Yamasaki K, Kurimura M, Kasai T, Sagara M, Kodama T, Inoue K: Determination of physiological plasma pentraxin 3 (PTX3) levels in healthy populations. Clin. Chem. Lab. Med. 47, 471-477 (2009)

29. Zanetti M, Bosutti A, Ferreira C, Vinci P, Biolo G, Fonda M, Valente M, Cattin L, Guarnieri G, Barazzoni R: Circulating pentraxin 3 levels are higher in metabolic syndrome with subclinical atherosclerosis: evidence for association with atherogenic lipid profile. Clin. Exp. Med. 9, 243-248 (2009) 